\title{
INTERACTION OF TWO-LEVEL ATOMIC SYSTEM WITH A SINGLE- MODE RADIATION FIELD
}

\author{
T. HAKIOĞLU \\ Physics Department, Bilkent University, Ankara, 06533 Turkey
}

\begin{abstract}
The Dicke model is examined in the limit of large number of atoms and /or large number of excitations. Superfluous instabilities arising from the rotating wave approximation is examined and counterrotating terms are shown to be crucial for the correct dynamical evolution in these limits.
\end{abstract}

\section{Introduction}

In this article we will briefly introduce the main results of the problem of interaction of an atomic cluster with " $p$ " atoms with a single mode resonant radiation field in the limit when $p$ and/or the total number of excitations $n$ is large. The principal assumption in this model is that the atom is considered with only two energy levels interacting via photon exchange. The principle reason is that besides the well-observed energy-momentum conservation in the observation time scales, atomic dipole transition between different energy levels are restricted by certain selection rules conserving the total angular momentum and parity due to the vector nature of the interaction. At the zero'th order the atom field interaction is resonant and is described by a two-level transition. The transition between these two levels takes place by the absorption or emission of a single light quantum with an energy exactly matching the energy difference of the two levels in question. The corrections to zero'th order approximation come from the spontaneous radiative processes and Lamb shift, atomic thermal collisions, recoiling effects and Doppler shift. The spontaneous radiative corrections produce a shift $\delta \nu / \nu \sim T / \tau \sim 10^{-3} \mathrm{eV}$ where $T \sim 10^{-15} \mathrm{~s}$ is a typical period of the radiation field and $\tau \sim 10^{-12} s$ is a typical lifetime for atomic energy scales. These corrections can grow as the number of energy levels in the atom gets larger nevertheless it can still be considered as a perturbative correction[1]. The nonrelativistic Doppler shift in the frequency of radiation for a gas of particles of mass $M$ and temperature $T$ is given in natural units by $\delta \nu / \nu=7.1610^{-7}(T / M)^{1 / 2}$. For a typical example of sodium atom at room temperature one finds $\delta \nu / \nu \simeq 10^{-13}$ which produces a negligible effect. Under more drastic Doppler shifts the Doppler-free spectroscopy can achieve resolutions approaching 1 part in $10^{11}$ which practically eliminates this effect.

It is clear that the zero'th order approximation is sufficient for most simple atoms except for pure quantum radiative processes, experimental techniques are available to 
suppress higher order effects by shifting them to negligible scales. We will not dwell on the details of the physical justification of two level systems longer and recommend to the reader a good survey by Allen and Eberly[2]. We will now briefly describe our model.

\section{The Dicke Model}

The simplifications made by the two level atom and a single field mode certainly pays back. In 1954 Dicke introduced a model to study the collective emission-absorption properties of a cluster of two-level atoms[3]. The major additional assumption in this model is that the linear size (i.e. $\mathcal{V}^{1 / 3}$ where $\mathcal{V}$ describes the volume occupied) of the cluster is much smaller than the wavelenght $\lambda$ of the emitted and absorbed radiation. Since $\mathcal{V}^{1 / 3} \ll \lambda$ the cluster-field coupling can be treated as a point-like interaction and all atoms within the cluster interact in phase with the same field strenght. This corresponds to the so called equivalent mode approximation and the atomic cluster is then composed of $p$ two-level indistinguishable dipoles which interact via exchange of quantum of radiation. Each atom is then represented by the complete set of "spin $=1 / 2$ " dipole operators,

$$
\begin{aligned}
\hat{L}_{j}^{(-)} & =|d\rangle_{j}{ }_{j}\langle u|, \\
\hat{L}_{j}^{(+)} & =|u\rangle_{j}{ }_{j}\langle d|, \\
\hat{L}_{j}^{(z)} & =\frac{1}{2}\left(|u\rangle_{j} \quad{ }_{j}\langle u|-| d\rangle_{j}{ }_{j}\langle d|\right)
\end{aligned}
$$

Here $|u\rangle_{j}$ and $|d\rangle_{j}$ describe the electron states in the excited and ground states of the $j$ 'th atom respectively as shown in Fig. (1) below. Eq.'s(1) can be shown easily to satisfy the

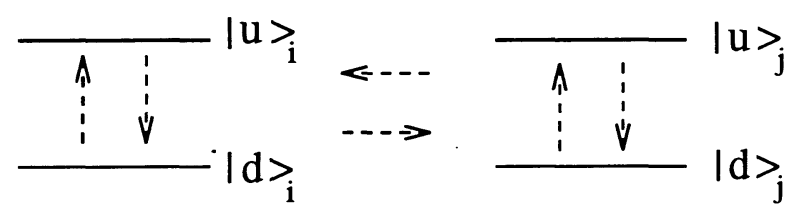

Figure 1. Two atoms in a Dicke cluster. Arrows indicate the exchange of photons.

$\mathrm{SU}(2)$ commutation relations,

$$
\begin{aligned}
{\left[\hat{L}_{i}^{(-)}, \hat{L}_{j}^{(+)}\right] } & =2 \hat{L}_{i}^{(z)} \delta_{i, j}, \\
{\left[\hat{L}_{i}^{( \pm)}, \hat{L}_{j}^{(z)}\right] } & =\mp \hat{L}_{i}^{( \pm)} \delta_{i, j} .
\end{aligned}
$$

Since dipoles at each atom are assumed not to couple by direct overlap of the electron wavefunctions localized at each atom ${ }^{1}$, the total number of electrons $\hat{N}_{j}$ at each atom in the cluster is separately conserved which is related to the total spin $\hat{L}_{j}^{2}$ where,

$$
\hat{N}_{i}=\left(|u\rangle_{(j) \quad(j)}\langle u|+| d\rangle_{(j) \quad(j)}\langle d|\right), \quad \text { and } \quad \hat{L}_{i}^{2}=\frac{\hat{N}_{i}}{2}\left(\frac{\hat{N}_{i}}{2}+1\right)
$$

\footnotetext{
${ }^{1}$ Collective effects in a model which inherently has the feature of electron hopping between localized atomic orbitals is an interesting and realistic model in certain cases. This extention will be studied in a separate publication.
} 
In the model we study each atom contains only one transition electron. An arbitrary microscopic state $|\ell\rangle_{c}$ of the cluster is then represented by,

$$
|\ell\rangle_{c}=\left|\ell_{z_{1}}, \ell_{z_{2}}, \ldots, \ell_{z_{n}}\right\rangle
$$

where $\ell_{z_{j}}= \pm 1 / 2(j=1, \ldots, n)$ is the eigenvalue of $\hat{L}_{j}^{z}$. If the linear size of the cluster is much smaller than the wavelenght of the dipole radiation we can apply the equivalent mode approximation. The whole cluster can then can be considered as a compound dipole with collective dipole operators,

$$
\begin{aligned}
\hat{\mathcal{L}}_{-} & =\sum_{j=1}^{N} L_{j}^{(-)}, \\
\hat{\mathcal{L}}_{+} & =\sum_{j=1}^{N} L_{j}^{(+)}, \\
\hat{\mathcal{L}}_{z} & =\sum_{j=1}^{N} L_{j}^{(z)},
\end{aligned}
$$

which also respect similar commutation relations à la Eq.'s(2) as,

$$
\begin{aligned}
& {\left[\hat{\mathcal{L}}_{-}, \hat{\mathcal{L}}_{+}\right]=2 \hat{\mathcal{L}}_{z},} \\
& {\left[\hat{\mathcal{L}}_{ \pm}, \hat{\mathcal{L}}_{z}\right]=\mp \hat{\mathcal{L}}_{ \pm} .}
\end{aligned}
$$

The algebra represented by these commutation relations permits us to find the macroscopic state of the cluster as linear superpositions of the microscopic ones in Eq.'s (4). Here one is tempted to adopt that the conserved quantum number under the action of the collective operators is the total number of atoms (or electrons) in the cluster $\hat{\mathcal{N}}$. The total cluster spin $\hat{\mathcal{L}}$ is then described by,

$$
\hat{\mathcal{N}}=\sum_{i=1}^{n} \hat{N}_{i}, \quad \text { and } \quad \hat{\mathcal{L}}^{2}=\frac{\hat{\mathcal{N}}}{2}\left(\frac{\hat{\mathcal{N}}}{2}+1\right)
$$

Let's adopt Eq.'s (7) temporarily and examine an arbitrary macroscopic state $|\ell m\rangle_{n}$ as a linear superposition of $|j\rangle_{n}$ as

$|\ell m\rangle_{n}=\sum_{j=1}^{n} c_{j}^{(n)}|j\rangle_{n} \quad$ with $\quad \hat{\mathcal{L}}_{z}|\ell m\rangle_{n}=m|\ell m\rangle_{n} \quad$ and $\quad \hat{\mathcal{L}}^{2}|\ell m\rangle_{n}=\ell(\ell+1)|\ell m\rangle_{n}$

where $|j\rangle_{n}$ describes a microscopic configuration and is nothing but Eq. (4) with $j$ atoms in the $u p($ i.e. $+1 / 2)$ and $n-j$ atoms in the down (i.e. $-1 / 2)$ spin configuration. A typical microscopic state is then,

$$
|j\rangle_{n} \Longleftrightarrow \mathcal{P}_{j}|\underbrace{+,+, \ldots,+}_{j} ; \overbrace{-,-, \ldots,-}^{n-j}\rangle
$$

where $\mathcal{P}_{j}$ describes any permutation over $j$ up and $n-j$ down spins. From Eq.'s (..) and (..) it is appealing to say that $-p / 2 \leq m=j-n / 2 \leq p / 2$ and $\ell=p / 2$. Although everything seems quite straightforward so far there is a subtlety involved such that the natural limit to the total spin $\ell$ is given by the total number of atoms hence $\ell_{\max }=p / 2$. However, in the most general case $\ell$ is a degree of freedom of the system and it is the natural limit for $m$ such that $-\ell \leq m \leq \ell$ and the total number of excited atoms under most general 
initial conditions may be less than the total number of atoms in the cluster. Another way of approach is that the total cluster spin $\ell$ is obtained by adding individual spins $\ell_{j}=1 / 2$ and therefore it is allowed to change between $p / 2$ and its minimum value, viz. $\ell=1 / 2$ for odd total number atoms or $\ell=0$ for even number of atoms. The value of $\ell$ is then fixed at the preparation of the initial macroscopic state and is a measure of cooperation between the atoms in their contribution to the radiative properties of the whole. Therefore in general we have $0 \leq \ell \leq p / 2$. In this case only those states with $-\ell+p / 2 \leq j \leq \ell+p / 2$ actively participate in the cooperative effects. Such states can in principle be uniquely determined in the initial state by proper choice of initial number of photons as well as the coefficients $c_{j}$ in Eq. (8).

Moreover the Dicke Hamiltonian is totally symmetric with respect to the exchange of indices of different atomic dipoles. This implies that the symmetrical or anti-symmetrical initial states never mix in their time evolution. Specific choice for the cooperation number can lead to distinct cooperative quantum effects of radiation. The reader can consult to a vast number of literature in this field of which only a few are listed in the references below $[1,2,3,4]$. Our specific aim in this section is to briefly investigate the most general case of arbitrary $p, \ell$ as well as total number of excitations $n$.

In this general case on has $-\ell \leq m \leq \ell$ with $\ell \leq p / 2$. Hence the non-vanishing matrix elements of the collective dipole operators for the arbitrary macroscopic state in Eq. (8) are given as (dropping the index $n$ from the $|\ell m\rangle$ states),

$$
\begin{aligned}
\left\langle\ell m\left|\hat{\mathcal{L}}_{z}\right| \ell m\right\rangle & =j-p / 2 \\
\left\langle\ell m+1\left|\hat{\mathcal{L}}_{+}\right| \ell m\right\rangle & =\sqrt{(\ell-j+p / 2)(\ell+j-p / 2+1)} \\
\left\langle\ell m-1\left|\hat{\mathcal{L}}_{-}\right| \ell m\right\rangle & =\sqrt{(\ell+j-p / 2)(\ell-j+p / 2+1)}
\end{aligned}
$$

We will use $|\ell m\rangle$ as the natural basis in the description of the atomic component of the generalized Dicke state.

The full Hamiltonian for the coupled cluster-single mode radiation state is given by,

$$
\hat{\mathcal{H}}=\omega\left(\hat{a}^{\dagger} \hat{a}+\frac{1}{2}\right)+\xi \hat{\mathcal{L}}_{z}+\gamma\left(\hat{a}^{\dagger} \hat{\mathcal{L}}_{-}+\hat{a} \hat{\mathcal{L}}_{+}\right)
$$

where $\omega$ is the frequency of the radiation, $\xi$ is the difference between the upper and lower atomic energy levels. Usually one considers $\omega=\xi+\Delta$ with $\Delta \neq 0$ describing the detuning from the exact resonance condition (i.e. $\Delta=0$ ).

\subsection{GENERAL SOLUTION}

The exact analytic solution of the Hamiltonian does not exist for an arbitrary number of atoms $p$ and/or arbitrary number of excitations $n$ in a closed form. The solution for arbitrary number of atoms with $n=1$ has been given by Cummings and Dorri[5] and Seke[6] a more general solution under the same conditions but including the retardation effects of the emitted radiation from each atom was considered earlier by Milonni and Knight[7]. For $p=1,2$ and arbitrary $n$ the exact solution is presented by Buz̀ek[8]. The solution of this simplified model is strong geometry dependent results. For arbitrary $p$ number of atoms initially with $n=1$ the radiation properties of the cluster can be varied from a superradiant (constructive interference) to subradiant (destructive interference). 
The case for $3 \leq p, n$ involves collective phenomena which are not present for the simple case when $p$ and/or $n$ are equal to one or two. A general pertubative approach for the $3 \leq p, n$ was formulated by Kozierowsky et al.[9]. Here we will examine the general $p$ and $n$ case primarily focusing on the qualitative aspects of the time evolution arising from the complexity of the eigen solutions.

In the Hamiltonian (11) the operator corresponding to the total number of excitations

$$
\hat{\mathcal{N}}=\hat{a}^{\dagger} \hat{a}+\hat{\mathcal{L}}_{z}+\frac{p}{2}
$$

since $[\hat{\mathcal{N}}, \hat{\mathcal{H}}]=0$ and the eigenvalue $n$ is an integral of motion. Since $\left[\hat{\mathcal{L}}^{2}, \hat{\mathcal{H}}\right]=0$ the cooperation number $\ell$ is also a good quantum number. We can restrict our attention onto a finite dimensional subspace of the Hilbert space corresponding to a given $n$. A typical state can then be represented at a particular instant by $r$ photons, $n-r$ atoms in the excited energy and $p-n+r$ atoms in the ground state energy level. Since $p$ is fixed and $n$ is determined by the initial conditions, depending on the $\ell$ values in question there are certain restrictions on possible values that $r$ can take. The conditions $|m| \leq \ell$ with $\ell \leq p / 2$ imply $-\ell+n-p / 2 \leq r \leq \ell+n-p / 2$ hence the total number of states $r$ can take is $2 \ell+1$. A dynamical state is then given in terms of combinations of $|n-r\rangle_{c} \otimes|r\rangle_{f}$ satisfying the above restrictions where the subscripts $c$ and $f$ denote the cluster and the field respectively. In order to find the matrix elements of the Hamiltonian, we thus need to replace $m=j-p / 2$ in Eq.'s $(10)$ by $m=n-r-p / 2$ and,

$$
\begin{aligned}
&\langle m-1|\otimes\langle r+1|\hat{\mathcal{H}}| r\rangle \otimes| m\rangle=\gamma v_{r} \\
&\langle m+1|\otimes\langle r-1|\hat{\mathcal{H}}| r\rangle \otimes| m\rangle= \gamma v_{r-1} \quad \text { where } \\
& v_{r}=\sqrt{r+1} \quad \sqrt{\ell+n-r-p / 2)(\ell-n+r+p / 2+1)}
\end{aligned}
$$

with $\quad r_{\min } \leq r \leq r_{\max } \quad$ and $\quad \ell \leq p / 2$.

The equations above represent the generalized case for arbitrary $p$ and $n$. Here $r_{\max }$ and $r_{\text {min }}$ determine the maximum allowable range for a given $\ell, n$ and $p$. In the most general case,

$$
r_{\text {max }}=\ell+n-p / 2 \quad \text { and } \quad r_{\min }= \begin{cases}-\ell+n-p / 2, & \text { and } \ell \leq n-p / 2 \\ 0 & \text { otherwise. }\end{cases}
$$

At this point we analyze several distinct situations. Since $r_{\min } \geq 0$ and $r_{\max } \leq n$ the number of possibilities for certain values of $n$ and $p$ can be classified in $n<p, p<n$ and $n=p$ as indicated in Table. 1 below. 


\begin{tabular}{|c|c|c|c|c|}
\hline \multirow{3}{*}{ a) } & \multirow{3}{*}{$\begin{array}{l}r_{\min }=0 \\
\text { and } \\
r_{\max }=n\end{array}$} & \multirow{3}{*}{$\ell=p / 2$} & $r_{\min }=0$ forced bound & $n<p$ \\
\hline & & & unphysical & $p<n$ \\
\hline & & & $r_{\min }=0$ natural bound & $n=p$ \\
\hline \multirow{3}{*}{ b) } & \multirow{3}{*}{$\begin{array}{l}r_{\min }=0 \\
\text { and } \\
r_{\max }<n\end{array}$} & \multirow{3}{*}{$\ell<p / 2$} & $\left\{\begin{array}{rr}r_{\min =0} \text { natural } & \text { if } \mathrm{n}>\mathrm{p} / 2 ; \\
\text { " forced } & \text { if } \mathrm{n}<\mathrm{p} / 2 .\end{array}\right.$ & $n<p$ \\
\hline & & & unphysical & $p<n$ \\
\hline & & & unphysical & $n=p$ \\
\hline \multirow{3}{*}{ c) } & \multirow{3}{*}{$\begin{array}{l}r_{\min }>0 \\
\text { and } \\
r_{\max }=n\end{array}$} & \multirow{3}{*}{$\ell=p / 2$} & unphysical & $n<p$ \\
\hline & & & allowed & $p<n$ \\
\hline & & & unphysical & $n=p$ \\
\hline \multirow{3}{*}{ d) } & \multirow{3}{*}{$\begin{array}{l}r_{\min }>0 \\
\text { and } \\
r_{\max }<n\end{array}$} & \multirow{3}{*}{$\ell<p / 2$} & $\begin{cases}\text { allowed } & \text { if } \mathrm{n}>\mathrm{p} / 2 ; \\
\text { unphysical } & \text { if } \mathrm{n}<\mathrm{p} / 2 .\end{cases}$ & $n<p$ \\
\hline & & & allowed & $p<n$ \\
\hline & & & allowed & $n=p$ \\
\hline
\end{tabular}

Table 1

It is crucial to remember that Dicke's superradiance condition $\ell=p / 2, m=0$ can be met initially within all the physically realizable parts $(a)$ and $(c)$ of Table. 1 . Dicke's subradiance can be realized within parts $(b)$ and $(c)$ if in the initial state $r_{\max }=n-p / 2$ which implies the $\ell=0, m=0$ singlet. The preparation of initial conditions in such a way that the time evolution will be dominated by superradiant or subradiant states is a difficult experimental task. First experiment on superradiant systems was performed in 1973 by Skribanowitz et al.[10] and that for subradiant states has been done in 1985 by Crubellier et al. and Pavolini et al.[11]. A good account on experimental realization of the required symmetry properties for partial (full) observation of superradiance/subradiance has been given in the former reference.

A different approach to subradiance has been suggested by Cummings[5] considering the spatial distribution of the atoms in the "equivalent mode" cluster for arbitrary $p$ as well as number of initial field modes when only one atom is initially excited. The underlying principle behin subradiance, whether it is prepared by a particular initial state or by randomly distributing atoms in the cluster is based on the principle of destructive interference. The very commonly studied case of $p$ atoms and $n=1$ with field initially in the vacuum state corresponds to the case $a$ in Table. 1 with $n<p$ and $n=p$. In this category, Cummings and Dorri[5] studied the evolution of an asymmetric initial state and for instance Seke[6] examined the symmetric case. The original Jaynes-Cummings model corresponds the case $p=1$ and arbitrary number of initial photons $r_{i n}>p$ and hence $n>p$. The photon number range is therefore given by $n-p \leq r \leq n$. This is contained in case $(c)$ in Table. 1 with $\ell=p / 2$ and hence $\ell=1 / 2$. Buz̀ek[8] also studied the consequences of the spatial distribution of atoms in the spatially extended cluster (i.e. linear size of the cluster is compatible with the wavelenght of the single resonant field). He considered the case of $p=2$ with $m=1$ or $m=2$ initially with an arbitrary number of photons in the initial field state. Here for the number of initial photons $2<r_{i n}$ hence $2<n 2$ and thus $n-2 \leq r \leq n$ which can be found within case $(c)$ in Table. 1. Since in Buz̀ek's calculation $\ell=p / 2$ and thus only $\ell=1$ is allowed. He also examined the case where the initial field is in a coherent state again with $p=2$. For this case each Fock component with a specific 
number of photons (i.e. $r$ ) can be studied independently. Nevertheless, since for each such component it is true that $n-p \leq r \leq n$ we still have the case $(c)$ corresponding to $\ell=p / 2$ yielding $\ell=1$ for $p<n$. For those components with $n<p$ or $n=p$ case $(c)$ is unphysical and thus we must have case $a$ but still with $\ell=1$.

Recently Kozierowski et al.[9] considered systems with three and more excited atoms in the initial state for arbitrary $p$. In such systems with $n \geq 3$ super structures modulating the zero'th order collapses and revivals appear arising from the non-equidistant eigenvalue distribution. In their exact results Kozierowski et al. considered the case with $n=3$ with arbitrary $p$ also using symmetric wavefuntions with $0 \leq r \leq n$. Again, this corresponds to the case $(a)$ in Table. 1 with $\ell=p / 2$.

Hence we can see that, for symmetric initial states, or for all cases when $r_{\max }=n$ the cooperation number does not need to be mentioned since in this case it is directly implied that $\ell=p / 2$. For $\ell=p / 2$ Eq.'s (13) can be seen to reduce into a relatively more conventional form via $^{2}$

$$
v_{r}=\sqrt{r+1} \sqrt{(n-r)(p-n+r+1)} .
$$

We can then write the Hamiltonian in Eq. (11) as

$$
\hat{\mathcal{H}}=\omega\left(\hat{\mathcal{N}}+\frac{1}{2}\right)-\Delta\left(\hat{a}^{\dagger} \hat{a}+1 / 2\right)+\gamma\left(\hat{a}^{\dagger} \hat{\mathcal{L}}_{-}+\hat{a} \hat{\mathcal{L}}_{+}\right)
$$

We will now rescale the matrix coefficients so that $\gamma$ is implicitly unity. The resulting matrix to be diagonalized is given by,

$$
\left(\begin{array}{cccccc}
-\epsilon-\Delta m_{\max } & v_{r_{\min }} & 0 & \ldots & \ldots & 0 \\
v_{r_{\min }} & -\epsilon-\Delta\left(m_{\max }-1\right) & v_{r_{\min }+1} & \ldots & 0 \ldots & 0 \\
0 & v_{r_{\min }+1} & \ldots & \ldots & \ldots & 0 \\
\vdots & \vdots & \vdots & \vdots & -\epsilon-\Delta\left(m_{\min }+1\right) & v_{r_{\max }} \\
0 & \ldots & \ldots & 0 & v_{r_{\max }} & -\epsilon-\Delta m_{\min }
\end{array}\right)
$$

where $m_{\max }=n-p / 2-r_{\min }$ and $m_{\min }=n-p / 2-r_{\max }$. Denoting the eigenvalues by $\epsilon^{(s)}$ and eigenvectors by $\left.\psi\right\rangle^{(s)}$ where $1 \leq s \leq n+1$ is the eigenvalue index, we have for $n=1$ and arbitrary $p$,

$$
\begin{aligned}
& \left.\epsilon_{1}=\frac{1}{2}\left[(p-1) \Delta+\sqrt{\Delta^{2}+4 v_{0}^{2}}\right] \quad \Rightarrow|\psi\rangle_{1}=\left(\frac{4 v_{0}^{2}}{\Delta^{2}+8 v_{0}^{2}}\right)^{1 / 2}|0\rangle+\left(\frac{\Delta^{2}+4 v_{0}^{2}}{\Delta^{2}+8 v_{0}^{2}}\right)^{1 / 2}|1\rangle\right) \\
& \left.\epsilon_{2}=\frac{1}{2}\left[(p-1) \Delta-\sqrt{\Delta^{2}+4 v_{0}^{2}}\right] \quad \Rightarrow \quad|\psi\rangle_{2}=\left(\frac{\Delta^{2}+4 v_{0}^{2}}{\Delta^{2}+8 v_{0}^{2}}\right)^{1 / 2}|0\rangle-\left(\frac{4 v_{0}^{2}}{\Delta^{2}+8 v_{0}^{2}}\right)^{1 / 2}|1\rangle\right)
\end{aligned}
$$

where $v_{0}=\sqrt{(\ell+1-p / 2)(\ell+p / 2)}$. For $p=1$ and $n$ arbitrary we have the case $(c)$ in Table. (1). The eigenvalues and eigenvectors are

$$
\begin{array}{llll}
\epsilon_{1}=\sqrt{\Delta^{2} / 4+n} & \Rightarrow & |\psi\rangle_{1}=\left(\sqrt{1-A^{2}}|n-1\rangle+A|n\rangle\right) \\
\epsilon_{2}=-\epsilon^{(1)} & \Rightarrow & |\psi\rangle_{2}=\left(A|n-1\rangle-\sqrt{1-A^{2}}|n\rangle\right)
\end{array}
$$

${ }^{2}$ Eq. (15) is more appropriate and simple for the case $n \leq p$. For the opposite case its symmetric equivalent with $v_{\tilde{r}}=\sqrt{\tilde{r}+1} \sqrt{(p-\tilde{r})(n-p+\tilde{r}+1)}$ with $0 \leq \tilde{r} \leq p$ and $\tilde{r}=r+(p-n)$ is more appropriate to use. In this case there are $p+1$ eigenvalues as opposed to $n+1$ in the former case. 
where $A=(1+\sin \alpha) / \sqrt{2}$ with $\sin \alpha=\frac{\Delta}{2} / \sqrt{\Delta^{2} / 4+n}$. For $p=2$ and $n$ arbitrary we can have $\ell=1$ or $\ell=0$. For the former $n-2 \leq r \leq n$ and we have the case (c) in Table. (1). Whereas for the latter (i.e. $\ell=0$ ) we have only one allowed photon number $r=n-1$ and this implies full radiation trapping for the antisymmetric initial state $|\ell=0 m=0\rangle$. For $\Delta \neq 0$ the eigenvalues and eigenvectors are not as simply expressed as in the $\Delta=0$ case. The eigenvalues and eigenvectors are, for $\ell=1$,

$$
\begin{array}{ll}
\epsilon_{1}=u+v, & \Rightarrow|\psi\rangle_{1}=A_{0}|n-2\rangle+A_{1}|n-1\rangle+A_{2}|n\rangle \\
\epsilon_{2}=u \cos \varphi+v \cos 2 \varphi, & \Rightarrow|\psi\rangle_{1}=B_{0}|n-2\rangle+B_{1}|n-1\rangle+B_{2}|n\rangle \\
\epsilon_{1}=u \cos 2 \varphi+v \cos \varphi, \quad \Rightarrow|\psi\rangle_{1}=C_{0}|n-2\rangle+C_{1}|n-1\rangle+C_{2}|n\rangle
\end{array}
$$

where $u$ and $v$ are such that,

$$
\begin{aligned}
& u=\left\{\Delta+\sqrt{\Delta^{2}+\left(4 n-2+\Delta^{2}\right)^{3}}\right\}^{1 / 3} \\
& u=\left\{\Delta-\sqrt{\Delta^{2}+\left(4 n-2+\Delta^{2}\right)^{3}}\right\}^{1 / 3}
\end{aligned}
$$

with $\cos \varphi=-v /(2 u)$ and

$$
\begin{array}{lll}
A_{1}=\sqrt{\frac{\epsilon_{1}^{2}-\Delta^{2}}{\epsilon_{1}^{2}-\Delta^{2}+\epsilon_{1}(4 n-2)+2 \Delta}}, & A_{0}=-\frac{\sqrt{2 n-2}}{\epsilon_{1}-\Delta} A_{1}, & A_{2}=-\frac{\sqrt{2 n}}{\epsilon_{1}+\Delta} A_{1} \\
B_{1}=\sqrt{\frac{\left(\epsilon_{2}\right)^{2}-\Delta^{2}}{\left(\epsilon_{2}\right)^{2}-\Delta^{2}+\epsilon_{2}(4 n-2)+2 \Delta}}, & B_{0}=-\frac{\sqrt{2 n-2}}{\epsilon_{2}+\Delta} B_{1}, & B_{2}=-\frac{\sqrt{2 n}}{\epsilon_{2}-\Delta} B_{1} \\
C_{1}=-B_{1}, & C_{0}=B_{0}, & C_{2}=B_{2}
\end{array}
$$

where $|r\rangle=|n-r\rangle_{a} \otimes|r\rangle_{f}$. For $\ell=0$ we have a zero mode with

$\left|\psi_{0}\right\rangle=|\ell=0 m=0\rangle=\frac{1}{2}(|+-\rangle-|-+\rangle) \otimes|n-1\rangle$. For $n<p$ the photon number is bounded by $0 \leq r \leq 2$ and thus we have the case (a) with $\ell=p / 2$. Whereas for $p<n$ the photon number range is $1 \leq r \leq 2$ and this again indicates that $\ell=p / 2$ which corresponds to the case $(c)$. The corresponding eigenvalues and eigenvectors are obtained from the symmetric case by interchanging $n$ and $p$ in Eq.'s (21) and (22).

We can also briefly mention the results for $n=3$ and $n=4$ for arbitrary $p$. The $n=3$ case with arbitrary $p$ was solved by Kozierowski[9] using symmetric initial states. For $p$ and $n$ being small and primarily less than two the eigenfrequencies are commensurate and the time dependence of the fluctuating observables are given by regular oscillations. Starting from $3 \leq n$ or $3 \leq p$ the eigenfrequencies become incommensurate as illustrated above. This is reflected on the time dependence of the atomic population inversion and incommensurate overtones of the eigenfrequencies manifest themselves in the appearance of superimposed modulations on the envelope function.

\subsubsection{Commensurate versus Incommensurate regimes}

If a number of frequencies $\epsilon^{(f)}$ where $f \leq(n+1)$ are incommensurate then for a set of integers $s_{1}, s_{2}, \ldots, s_{n}$ the frequency sum

$$
s_{1} \epsilon^{(1)}+s_{2} \epsilon^{(2)}+\ldots+s_{f} \epsilon^{(f)}+\ldots+s_{n} \epsilon^{(n)}=0
$$


can not have a non-trivial solution except $s_{1}=s_{2}=\ldots=s_{n}=0$. This condition can be expressed equivalently by stating that there is at least one frequency $\epsilon^{(f)}$ of which ratio to all other frequencies is an irrational number. The representation of a dynamical observable $G(t)$ in the frequency spectrum

$$
\tilde{g}(\omega)=\int_{-\infty}^{\infty} d t e^{i \omega t} G(t)
$$

can display irregular behaviour since

$$
\tilde{g}(\omega)=\sum_{s_{1}, s_{2}, \ldots, s_{n}} \Gamma_{s_{1}, s_{2}, \ldots, s_{n}} \delta\left(\omega-\left[s_{1} \epsilon^{(1)}+s_{2} \epsilon^{(2)}+\ldots+s_{n} \epsilon^{(n)}\right]\right) .
$$

where in each summation $-\infty<s_{i}<\infty \quad(i=1,2, \ldots, n)$. By investigating the $|\tilde{g}(\omega)|$ one can determine the nature (i.e. periodic, quasi-periodic, irregular) of its phase space attractor. In Fig. (2) below the ratio of the smaller eigenfrequencies to the largest one is plotted for fixed values of $n$ ranging from 3 to 100 with respect to increasing values of $p$ for $n \leq p$ and for $\Delta=0$. The commensurability of the frequencies is not guaranteed at all parameter values although in the limit $1 \ll p / n$ the ratio of the frequencies approach to rational numbers. The opposite case with $p / n \ll 1$ is symmetric since it can always be mapped into an equivalent $1 \ll p / n$ case by a simple shift in the photon number (see the footnote on page 9). Therefore, the strongest incommensuration in Fig. (2) corresponds to $n=p$. Continuous detuning from resonance is a controlled nevertheless nontrivial way of
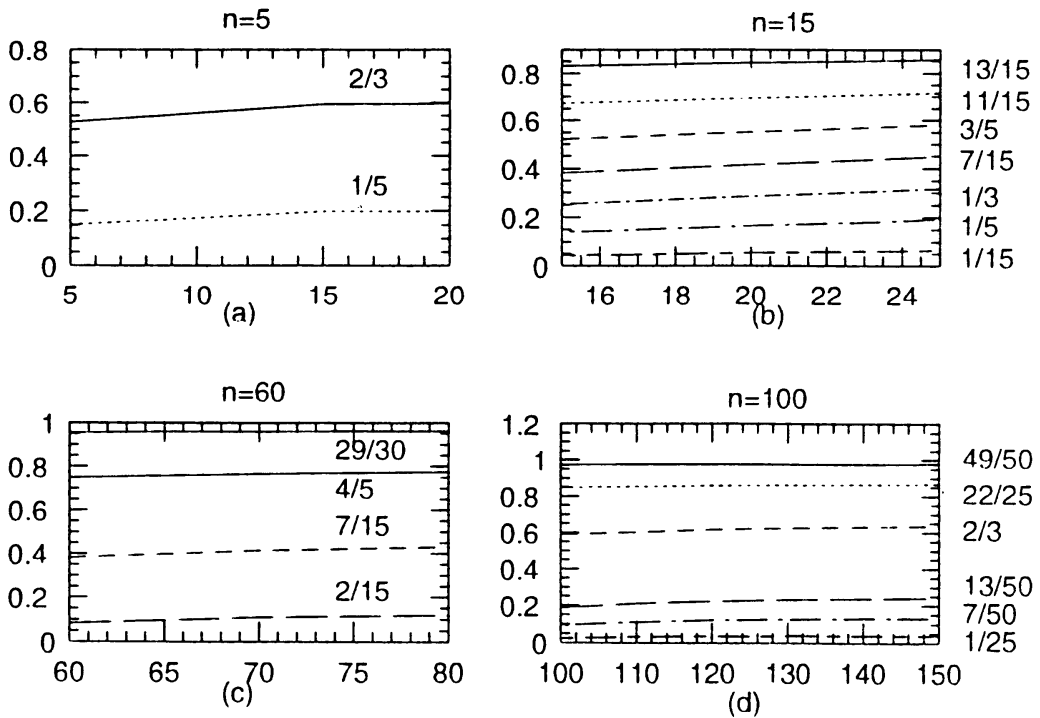

Figure 2. The ratio of the smaller eigenvalues to the largest one (i.e. $\left.\epsilon_{n, p}^{(j)} / \epsilon_{n, p}^{\max }\right)$ as $n \leq p$ varies. a) $n=5$ and from bottom to top $j=5$ and $j=3$; b) $n=15$ and from bottom to top $j=15,13,11,9,7,5,3$; c) $n=60$ and from bottom to top $j=53,33,13,3$; d) $n=100$ and from bottom to top $j=97,87,75,35,13,3$.

observing incommensuration. Non-zero detuning slightly softens the eigenfrequencies and increases their non-linear dependence on $n$ and $p$. Strong nonlinearity is observed when 
$n$ and $p$ are close to each other. The increase in the allowed range for photon number $r_{\text {max }}-r_{\text {min }}=2 \ell+1$ also increases the effect.

Incommensurate frequencies are easily established for a number of parameter values. For instance for $n=3$ and $n=4$ at $\Delta=0$ we list a few cases for which one obtains irrational values for the frequency ratios out of many other possible ones ${ }^{3}$

\begin{tabular}{l|l|l}
$n$ & $p$ & $\epsilon^{(1)} / \epsilon^{(3)}$ \\
\hline 3 & 3 & $\sqrt{\frac{10-\sqrt{73}}{10+\sqrt{73}}}$ \\
\hline 3 & 4 & $\sqrt{\frac{15-\sqrt{153}}{15+\sqrt{153}}}$ \\
\hline 4 & 4 & $\sqrt{\frac{25-3 \sqrt{33}}{25+3 \sqrt{33}}}$ \\
\hline 4 & 5 & $\sqrt{\frac{33-\sqrt{553}}{33+\sqrt{553}}}$ \\
\hline
\end{tabular}

\begin{tabular}{l|l|l}
$n$ & $p$ & $\epsilon^{(1)} / \epsilon^{(3)}$ \\
\hline 4 & 6 & $\sqrt{\frac{30-4 \sqrt{89}}{30+4 \sqrt{89}}}$ \\
\hline 4 & 7 & $\sqrt{\frac{53-\sqrt{1201}}{53+\sqrt{1201}}}$ \\
\hline 4 & 8 & $\sqrt{\frac{65-3 \sqrt{177}}{65+3 \sqrt{177}}}$ \\
\hline 4 & 9 & $\sqrt{\frac{75-3 \sqrt{233}}{75+3 \sqrt{233}}}$ \\
\hline
\end{tabular}

Table. 2

Perhaps a more illustrative quantity to examine is the time evolution of the various moments of the photon number $m^{(k)}(t)=\left\langle\left(\hat{a}^{\dagger} \hat{a}\right)^{k}\right\rangle$ as the dynamical observable replacing the role of $\mathcal{G}(t)$ in Eq. $(24)$.

Here there is a competition between various time scales involved. If the eigenfrequencies are labeled as $\epsilon_{[n / 2+1]}^{n, p} \leq \epsilon_{[n / 2]}^{n, p} \leq \epsilon_{[n / 2-1]}^{n, p} \cdots \leq \epsilon_{2}^{n, p} \leq \epsilon_{1}^{n, p}$ with $[x]$ representing the integer part of " $x$ ", the smallest finite period is given by

$$
\begin{array}{ll}
T_{1}^{n, p}=2 \pi /\left(\epsilon_{1}^{n, p}-\epsilon_{[n / 2+1]}^{n, p}\right), & \text { if } \quad \mathrm{n} \text { is odd } \\
T_{1}^{n, p}=2 \pi /\left(\epsilon_{1}^{n, p}-\epsilon_{n / 2}^{n, p}\right), & \text { if } \quad \mathrm{n} \text { is even }
\end{array}
$$

with the main difference arising from the fact that for $n=e v e n$ one eigenvalue is always zero yielding an infinite period. On the other the difference between the two smallest eigenfrequencies determine the period of revivals in the atomic population inversion and corresponds to the smallest beat frequency. The revival period is given by,

$$
\begin{array}{lll}
T_{n, p}^{(2)}=2 \pi /\left(\epsilon_{n, p}^{([n / 2])}-\epsilon_{n, p}^{([n / 2+1])}\right), & \text { if } & \mathrm{n} \text { is odd } \\
T_{n, p}^{(2)}=2 \pi /\left(\epsilon_{n, p}^{(n / 2)}-\epsilon_{n, p}^{n / 2-1)}\right), & \text { if } \mathrm{n} \text { is even }
\end{array}
$$

because of the same reason as above. Comparison of the time scales in (26) and (27) is crucial to understand the dynamical time correlations in $m^{(k)}(t)$. In Fig. (3) below, we examine the ratio $T_{1}^{n, n} / T_{2}^{n, n}$ for various $n$ values. As $n$ increases $T_{1}^{n, n} / T_{2}^{n, n}$ tends to zero. In the case of arbitrary $n<p$ the same qualitative behaviour is observed.

${ }^{3}$ For the $n=3$ and $n=4$ cases the frequency ratios can be represented as $\sqrt{\frac{a+b \sqrt{c}}{a-b \sqrt{c}}}$ where $a, b, c$ are positive integers. It can be shown that if $c$ does not divide $a^{2}$ and $b^{2}$ the frequency ratio is an irrational number. Such condition is satisfied when $c$ itself or its dividents are prime numbers. 


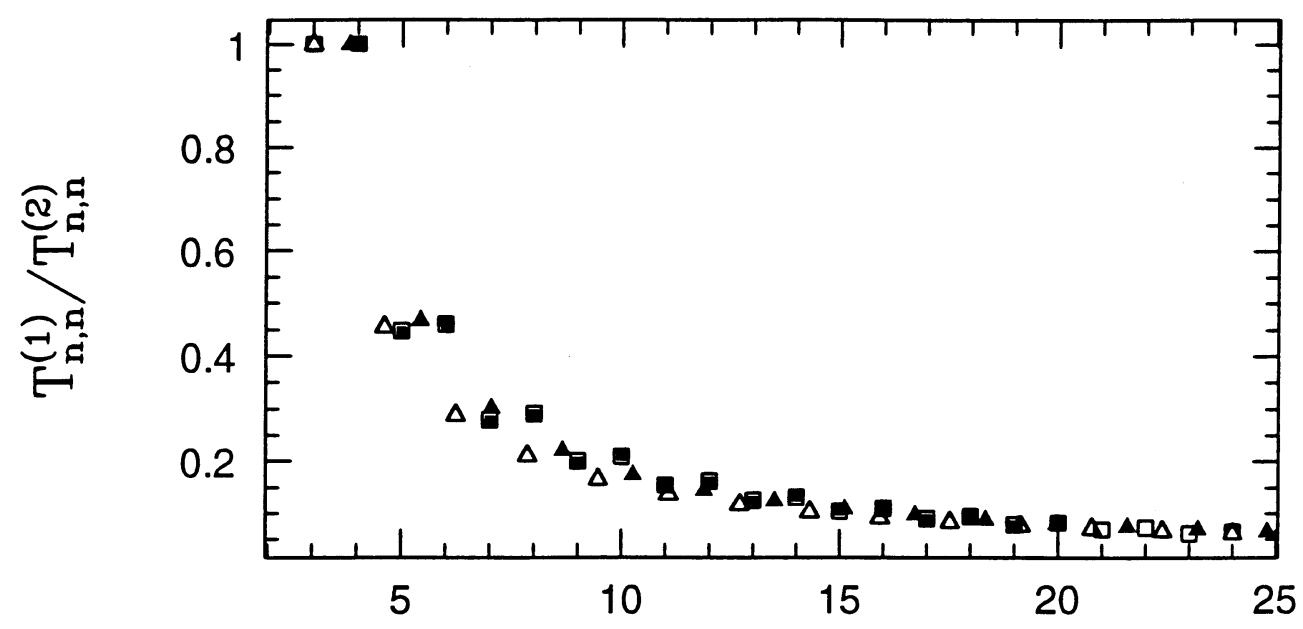

$\mathrm{n}$

Figure 3. Here the open and solid triangles indicate $n=p=o d d$, and $n=p=\epsilon v e n$ respectively for $\Delta=0$. The open and solid rectangles indicate the same for $\Delta=0.3$.

We can now examine the moments $m^{(k)}(t)$ and their correlations. The time correlations within a single revival (i.e. $T_{n, p}^{(1)} \leq t \leq T_{n, p}^{(2)}$ ) and those spanning a time interval large enough to intercorrelate more than one revivals (i.e. $T_{n, p}^{(1)} \leq T_{n, p}^{(2)} \leq t$ exhibit qualitatively different behaviour. In the Fig.'s (4) and (5) below $m^{(k)}(t)$ for $k=1,2,4,6$ are plotted. Fig.'s (4.a,b,c,d) and Fig.'s (4.e,f,g,h) represent $n=p=9$ and $n=6, p=9$ respectively. For the former the time dependence of all moments are irregular. The period of the envelope for the latter can be estimated using Kozierowski's perturbative result as[9],

$$
T_{R}=\frac{8 \pi}{15}(p-n / 2+0.5)^{3 / 2} \simeq 28
$$

which are within ten percent of the numerical calculations (for clarity two full periods are shown in the figure). In Fig.'s (5.a,b,c,d) and Fig.'s (5.e,f,g,h) below $n=p=40$ and $n=20, p=40$ are plotted. The same trend continues here and the time dependence of the $n=p$ case is erratic whereas collective collapses and revivals are observed for the $n=$ $p=40$ case. The revival period can be estimated from Eq. (28) above as $T_{R} \simeq 156$ which again underestimates the revival period by about ten percent. An interesting observation here is that during the short collapse period of the mean photon number there are strong fluctuations in the number of photons as visible in the higher moments $m^{(k)}(t)$ with $k>1$ (also note the scale change as $k$ increases). In Fig.'s (6) we numerically confirm for $\Delta=$ 0.3 the effect of non-zero detuning driving the system into a strong incommensuration. Fig. (6.a,b,c,d) represents $n=p=40$, whereas Fig. (6.e,f,g,h) represents $n=20, p=40$.

\section{Physical limitations in the large $n$ limit}

The exact solution of the eigen system in Eq.'s (16) and (17) has not been found in a closed analytic form. The eigenvalues of the full Hamiltonian are always symmetrically 

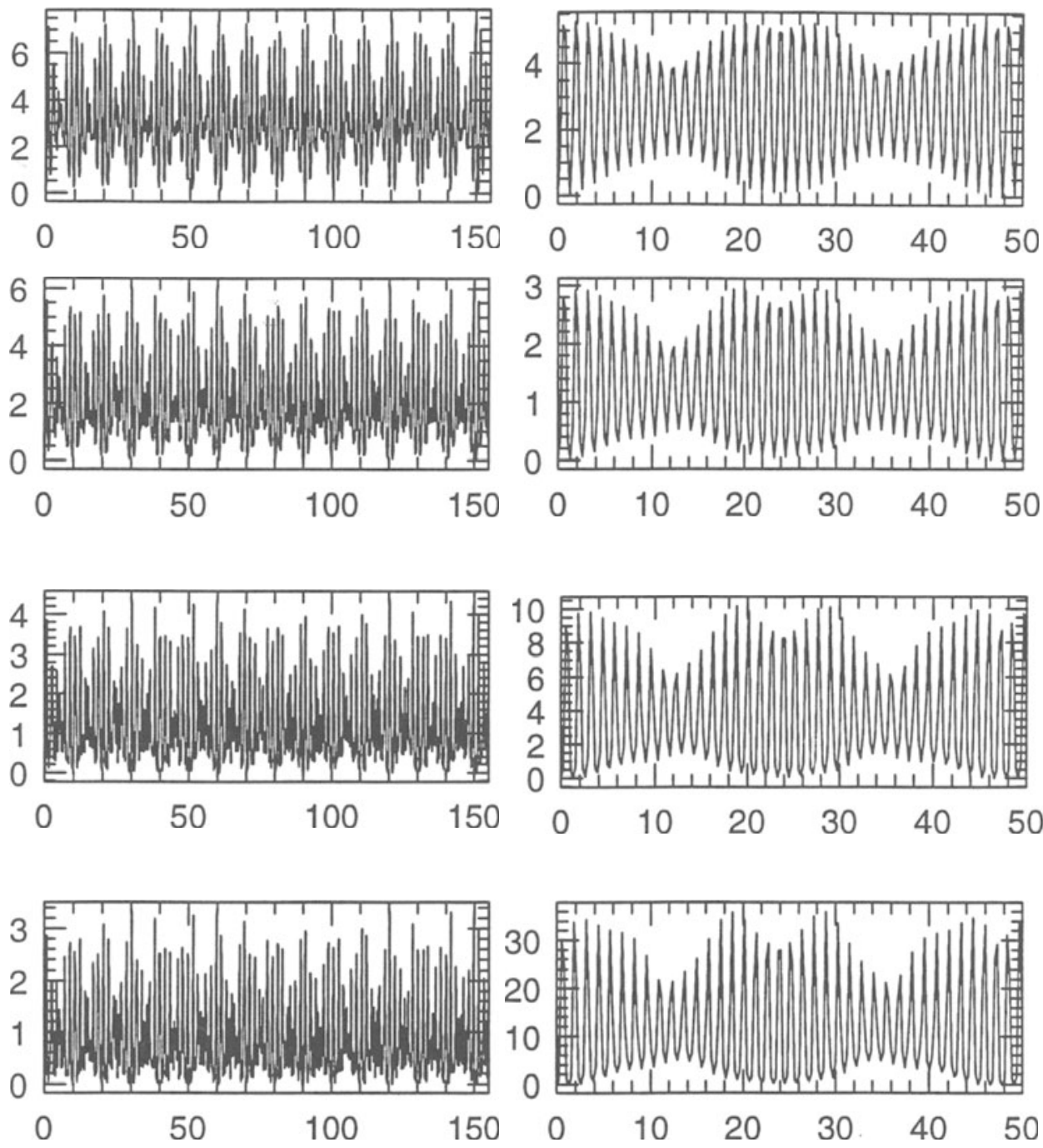

Figure 4. From the top left to the bottom left the plots are (a),(b),(c) and (d) representing $k=1,2.4,6$ respectively for $n=p=9$ and for $\Delta=0$. The vertical axes are scaled for $k=2$ with $x 10$, for $k=4$ with $x 10^{3}$ and for $k=6$ with $x 10^{5}$. On the right from top to bottom the plots (c), (d), (e) and (f) represent the same order in $k$ for $n=6 p=9$ and for $\Delta=0$. The vertical axes are scaled for $k=2$ with $x 10$, for $k=4$ with $x 10^{2}$ and for $k=6$ with $x 10^{3}$.

distributed around the central value $\omega n$ as $E_{j}^{(n)}=\omega n \pm \epsilon_{n}^{(j)}$. This is a strong signal that one should examine the large $n$ and/or $p$ limit with extra care. The behaviour of the $\epsilon_{n}^{(j)}$ 's as $n$ and/or $p$ increase can be examined using the recursive properties of the determinant. It has been shown previously[12] that the properties of eigensolutions of 

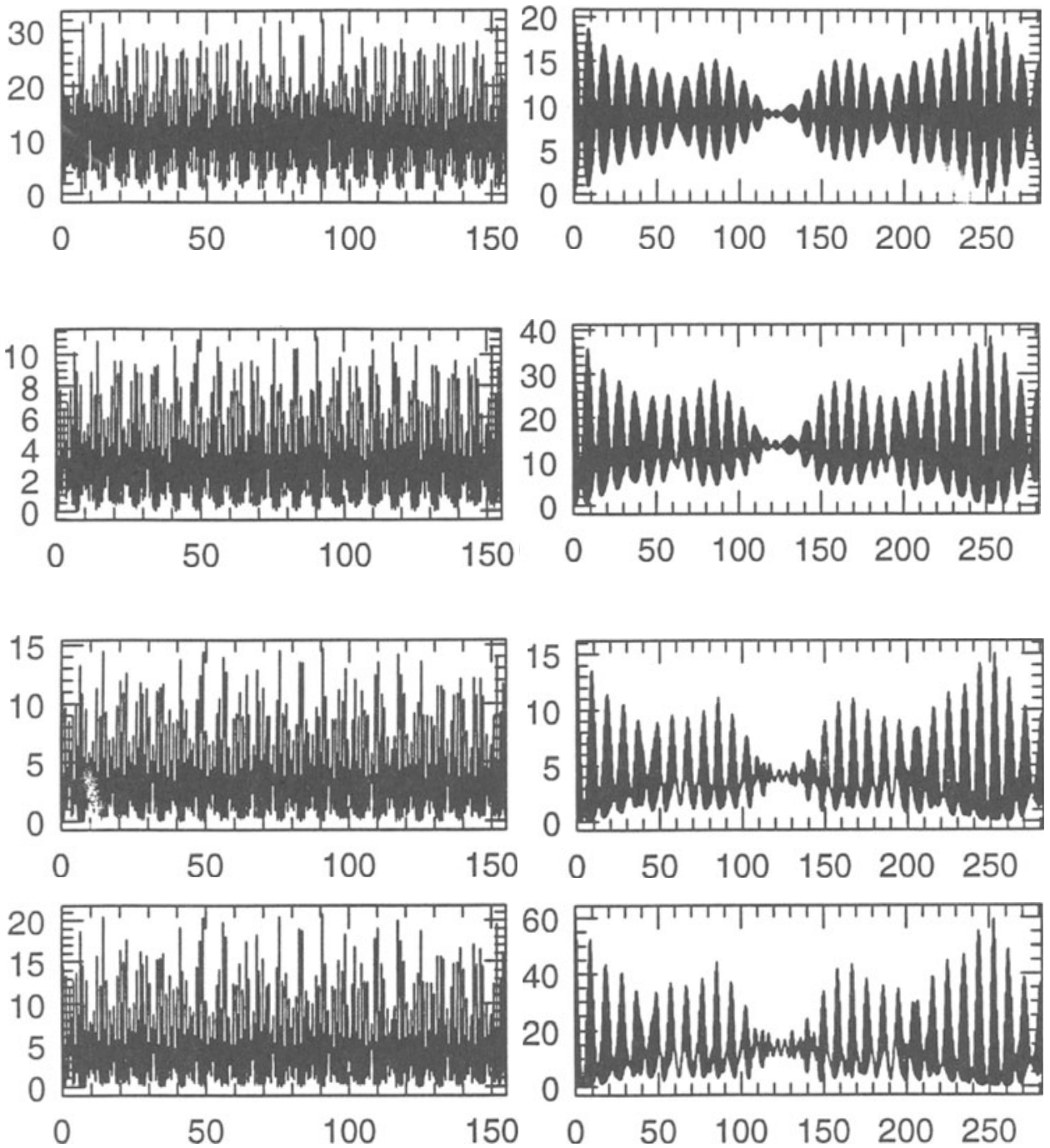

Figure 5. From the left top to the left bottom the plots are (a),(b),(c) and (d) representing $k=1.2,4,6$ respectively for $n=p=40$ and for $\Delta=0$. The vertical axes are scaled for $k=2$ with $x 10^{2}$, for $k=4$ with $x 10^{5}$ and for $k=6$ with $x 10^{8}$. On the right from top to bottom the plots (c), (d), (e) and (f) representing the same order in $k$ for $n=20 p=40$ and for $\Delta=0$. The vertical axes are scaled for $k=2$ with $x 10$, for $k=4$ with $x 10^{4}$ and for $k=6$ with $x 10^{6}$.

such simple Hamiltonians as (16) can be studied by orthogonal polinomials[13]. The model predicts that $\epsilon_{n}^{(j)}$ grow much faster than $\omega n$ eventually leading to negative energies. This is an artifact of the non-unitarity of the model in (16) arising from the assumption that the counterrotating terms are neligible. For instance the largest eigenvalue can be estimated 

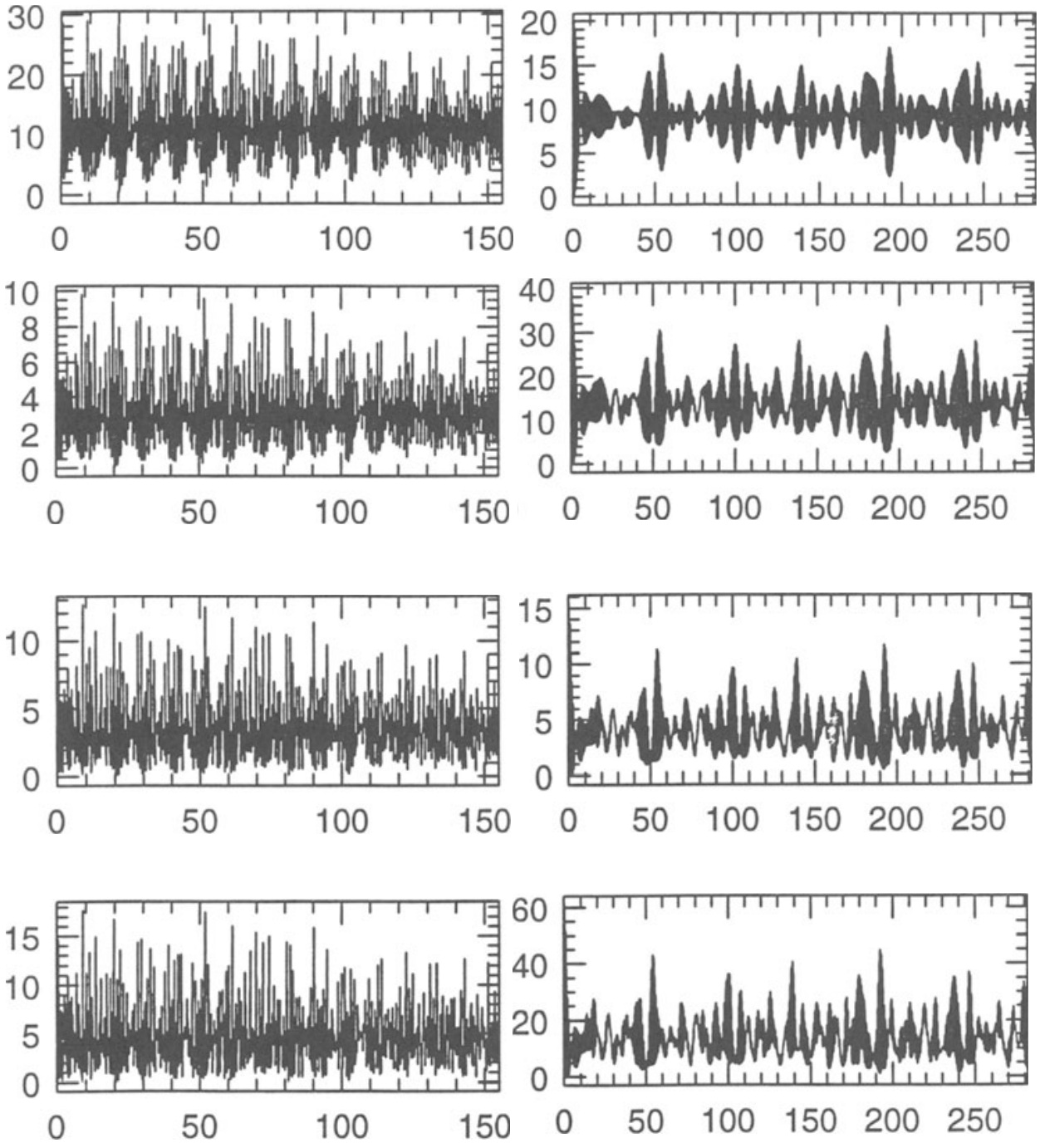

Figure 6. From the left top to the left bottom the plots are (a),(b),(c) and (d) representing $k=1,2,4,6$ respectively for $n=p=40$ and for $\Delta=0.3$. The vertical axes are scaled for $k=2$ with $x 10^{2}$, for $k=4$ with $x 10^{5}$ and for $k=6$ with $x 10^{8}$. On the right from top to bottom the plots (c), (d), (e) and (f) representing the same order in $k$ for $n=20 p=40$ and for $\Delta=0.3$. The vertical axes are scaled for $k=2$ with $x 10$, for $k=4$ with $x 10^{4}$ and for $k=6$ with $x 10^{6}$.

by the inequality $[12,13,14]$,

$$
\frac{1}{n} \sum_{r=0}^{n} v_{r}^{2} \leq \frac{1}{n} \sum_{r=0}^{n}\left(\epsilon_{r}^{n}\right)^{2} \leq\left(\left[\epsilon_{j}^{(n)}\right]_{\max }\right)^{2}
$$


the left hand side of the inequality can be computed from Eq. (15) which gives an approximate critical size for the cluster below which the rotating wave approximation can be used. Using (29), this critical size $p_{c}$ for the Hamiltonian in (16) is given by $p_{c}=3 \omega-2$ such that $p \ll p_{c}$ is required to secure the validity of the presented solution based on the rotating wave approximation. The formal solution including the counter rotating terms yields a unitary $S$-matrix but the solution itself can not be given in a simple form[15].

The counter rotating terms in the Hamiltonian are represented by $\hat{\mathcal{H}}^{\prime}=\gamma\left(\hat{a}^{\dagger} \hat{\mathcal{L}}_{+}+\right.$ $\left.\hat{a} \hat{\mathcal{L}}_{-}\right)$. The first order perturbative correction to the energy eigenvalues vanish. The second order correction can be calculated from ${ }^{4}$

$$
\epsilon_{2}^{i, n}=\sum_{i^{\prime}, n^{\prime}} \frac{\left\langle\left.\left\langle i^{\prime} n^{\prime}\left|\mathcal{H}^{\prime}\right| i n\right\rangle\right|^{2}\right.}{\epsilon_{0}^{i, n}-\epsilon_{0}^{i^{\prime}, n^{\prime}}} \quad i \leq(n+1) ; \quad i^{\prime} \leq\left(n^{\prime}+1\right)
$$

where only $n^{\prime}=n \pm 2$ contribute. Here it is important to know the full interaction strenght since the eigenvalues no more globally scale with the first power of the coupling constant $\gamma$. Eq. (30) is shown below for $n=p=20$ and for $\Delta=0$ and for $\Delta=0.3$ respectively. In the next table $n=p=10$ for the same $\Delta$ values are given (note that the second order corrections are given in units of $\gamma$ ).

The second order correction $\epsilon_{2}^{j, n}$ is consistently opposite in sign to the zero'th order eigenvalue $\epsilon_{0}^{j, n}$. The importance of this result is in the fact that corrections tend to confine the spreading eigenvalues for increasing $n$ and/or $p$. The net effect is to correct the superfluous instability beyond the critical region $p \sim p_{c}$ by pushing it to larger values. Additional details about the instability will be presented elsewhere[14].

${ }^{4}$ Since $\left[\hat{\mathcal{H}}^{\prime}, \hat{\mathcal{N}}\right] \neq 0$ different $n$ 's are mixed. In the Eq. $(29) \epsilon_{j}^{i, n}$ indicates the $j$ 'th order correction to the $i$ 'th eigenvalue of the unperturbed Hamiltonian (16) with a fixed $n$. 


\begin{tabular}{|c|c|c}
$j$ & $\epsilon_{0}^{j, n}$ & $\epsilon_{2}^{j, n} / \gamma$ \\
\hline 1 & 74.60 & -268.59 \\
\hline 2 & 65.52 & -226.38 \\
\hline 3 & 56.76 & -187.74 \\
\hline 4 & 48.34 & -152.61 \\
\hline 5 & 40.26 & -120.93 \\
\hline 6 & 32.56 & -92.64 \\
\hline 7 & 25.24 & -67.71 \\
\hline 8 & 18.34 & -46.12 \\
\hline 9 & 11.86 & -27.85 \\
\hline 10 & 5.79 & -12.77 \\
\hline 11 & 0.0 & 0.0 \\
\hline 12 & -74.60 & 268.59 \\
\hline 13 & -65.52 & 226.38 \\
\hline 14 & -56.76 & 187.74 \\
\hline 15 & -48.34 & 152.61 \\
\hline 16 & -40.26 & 120.93 \\
\hline 17 & -32.56 & 92.64 \\
\hline 18 & -25.24 & 67.71 \\
\hline 19 & -18.34 & 46.12 \\
\hline 20 & -11.86 & 27.85 \\
\hline 21 & -5.79 & 12.77 \\
\hline & $\Delta=0$ \\
\hline 15
\end{tabular}

\begin{tabular}{|l|l|l}
$j$ & $\epsilon_{0}^{j, n}$ & $\epsilon_{2}^{j, n} / \gamma$ \\
\hline 1 & 78.66 & -243.07 \\
\hline 2 & 69.40 & -200.56 \\
\hline 3 & 60.44 & -161.82 \\
\hline 4 & 51.79 & -126.95 \\
\hline 5 & 43.44 & -96.12 \\
\hline 6 & 35.43 & -69.35 \\
\hline 7 & 27.79 & -46.46 \\
\hline 8 & 20.57 & -27.12 \\
\hline 9 & 13.81 & -10.85 \\
\hline 10 & 7.52 & -3.13 \\
\hline 11 & 1.62 & -1.50 \\
\hline 12 & -4.12 & 1.22 \\
\hline 13 & -10.01 & 42.05 \\
\hline 14 & -16.21 & 63.38 \\
\hline 15 & -22.78 & 88.69 \\
\hline 16 & -29.75 & 116.91 \\
\hline 17 & -37.14 & 147.32 \\
\hline 18 & -44.95 & 180.06 \\
\hline 19 & -53.15 & 215.72 \\
\hline 20 & -61.72 & 254.76 \\
\hline 21 & -70.63 & 297.35 \\
\hline & $\Delta=0.3$ \\
\hline
\end{tabular}

Table 3. Corrections to the eigenenergies for $n=p=20$. 


\begin{tabular}{|c|c|c}
\hline$j$ & $\epsilon_{0}^{j, n}$ & $\epsilon_{2}^{j, n} \gamma$ \\
\hline 1 & 39.72 & -71.41 \\
\hline 2 & 31.43 & -53.48 \\
\hline 3 & 23.35 & -37.82 \\
\hline 4 & 15.46 & -24.04 \\
\hline 5 & 7.69 & -11.65 \\
\hline 6 & 0.0 & 0.0 \\
\hline 7 & -7.69 & 11.65 \\
\hline 8 & -15.46 & 24.04 \\
\hline 9 & -23.35 & 37.82 \\
\hline 10 & -31.43 & 53.48 \\
\hline 11 & -39.72 & 71.41 \\
\hline & \multicolumn{2}{|c|}{$\Delta=0$}
\end{tabular}

\begin{tabular}{|l|l|l}
$j$ & $\epsilon_{0}^{j, n}$ & $\epsilon_{2}^{j, n} / \gamma$ \\
\hline 1 & 41.59 & -64.53 \\
\hline 2 & 33.12 & -45.58 \\
\hline 3 & 24.81 & -29.55 \\
\hline 4 & 16.64 & -15.97 \\
\hline 5 & 8.64 & -1.53 \\
\hline 6 & 0.84 & -0.82 \\
\hline 7 & -6.77 & 14.19 \\
\hline 8 & -14.30 & 28.69 \\
\hline 9 & -21.91 & 44.03 \\
\hline 10 & -29.77 & 60.10 \\
\hline 11 & -37.90 & 77.78 \\
\hline & \multicolumn{3}{|c}{$\Delta=0.3$}
\end{tabular}

Table 3. Corrections to the eigenenergies for $n=p=10$

\section{Acknowledgements}

The author is gratefull to Dr. A. Miranowicz with whom most parts of this work were discussed during his visit at Bilkent.

\section{References}

1. The recent book by L. Mandel and E. Wolf is an excellent source for the treatment of spontaneous processes in two level systems. See Leonard Mandel and Emil Wolf, Optical Coherence and Quantum Optics (Cambridge University Press, 1995).

2. L. Allen and J.H. Eberly, Optical Resonance and Two-Level Atoms, Dover Publications, (New York 1975).

3. R.H. Dicke, Phys. Rev. 93, 99 (1954).

4. Nicholas E. Rehler and Joseph H. Eberly, Phys. Rev. A 3, 1735 (1970); G.S. Agarwal, Phys. Rev A 2, 2038 (1970); R. H. Lehmberg, Phy. Rev. A 2, 883 (1970); ibid , 889 (1970).

5. F.W. Cummings and A. Dorri, Phys. Rev. A 28, 2282 (1983); F.W. Cummings, Phys. Rev.Lett. 54, 2329 (1985).

6. J. Seke, Phys. Rev. A 33, 739 (1986).

7. W. Milonni and P. Knight, Phys. Rev. A 10, 1096 (1974).

8. V. Buz̀ek, Z. Phys. D 17, 91 (1990).

9. M. Kozierowski, A.A. Mamedov and S.M. Chumakov, Phys. Rev. A 42, 1762 (1990); M. Kozierowski and S.M. Chumakov, Phys. Rev. A 52, 4194 (1995).

10. N. Skribanowitz, I.P. Herman, J.C. McGillivray and M.S. Feld, Phys. Rev. Lett. 30, 309 (1973). 
11. A. Crubellier, S. Liberman, D. Pavolini and P. Pillet, J. Phys. B 18, 3811 (1985); D. Pavolini, A. Crubellier, P. Pillet, L. Cabaret and S. Liberman, Phys. Rev. Lett. 54, 1917 (1985).

12. Y. Orlov and V.V. Vedenyapin, Mod. Phys. Lett. B 9, 291 (1995).

13. Gabor Szegö, Orthogonal Polinomials, (American Mathematical Society, (1939).

14. T. Hakioğlu and A. Miranowicz, unpublished (1996).

15. Ricardo Ramirez and Miguel Orszag, J. Math. Phys. 22, 1306 (1980). 